\title{
REVIEW
}

\section{Pathology and clinical relevance of radial scars: a review}

\author{
M Kennedy, A V Masterson, M Kerin, F Flanagan
}

J Clin Pathol 2003;56:721-724

Radial scar (RS) is a benign, well recognised, radiological and pathological entity. Histologically, it is characterised by a fibroelastotic core with entrapped ducts and surrounding radiating ducts and lobules. Postmortem studies indicate that these lesions are present commonly in the population, especially in association with benign breast disease. In recent years, their clinical relevance has assumed more importance with the introduction of population based screening programmes. The exact pathogenesis of RS is unknown. Accumulating evidence indicates that they are associated with atypia and/or malignancy and, in addition, may be an independent risk factor for the development of carcinoma in either breast. In view of the association with atypia and malignancy, excision biopsy is justified in RS, although it has been argued that core biopsy evaluation and surveillance may be appropriate in selected patients.

See end of article for authors' affiliations ..................

Correspondence to: Dr M Kennedy, Department of Pathology, Mater Misericordiae University Hospital, Eccles St, Dublin 7, Ireland; mkennedy@mater.ie

Accepted for publication 15 May 2003
$\mathrm{R}$ adial scar (RS), also known as complex sclerosing lesion, is a well recognised radiological and pathological (although not necessarily synonymous) entity. Historically, RS has been referred to by several different terms, including sclerosing papillary proliferation, ${ }^{1}$ infiltrating epitheliosis, ${ }^{2}$ indurative mastopathy, ${ }^{3}$ benign sclerosing ductal proliferation, ${ }^{4}$ and nonencapsulated sclerosing lesion. ${ }^{5}$ Although postmortem studies ${ }^{6-8}$ indicate that these lesions are

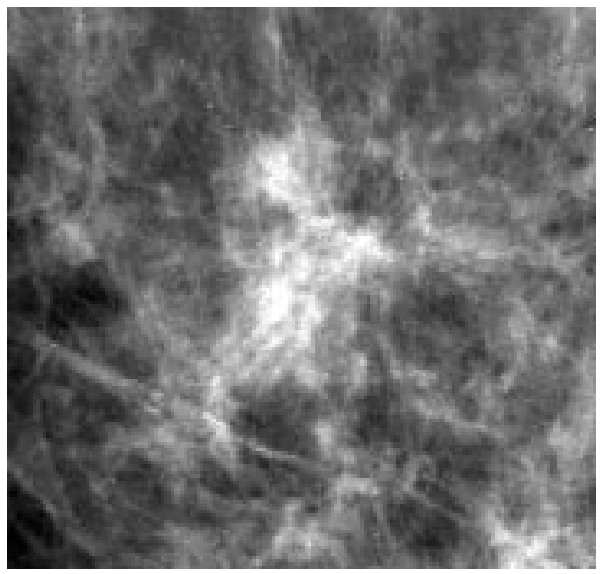

Figure 1 Characteristic mammographic appearance of radial scar. present commonly in the population, especially in association with benign breast disease, ${ }^{79}$ their clinical relevance has assumed more importance in recent years with the advent of population based screening programmes. RS are benign lesions, but accumulating evidence indicates that they are (1) associated with atypia and/or malignancy and (2) may be an independent risk factor for the development of carcinoma in either breast.

\section{RADIOLOGY}

The mammographic appearances of RS are well documented. They are characterised by an area of architectural distortion and are defined according to the criteria of Tabar and Dean ${ }^{11}$; namely, (1) the presence of a central radiolucency, (2) the presence of radiating long thin spicules, (3) varying appearance in different projections, (4) radiolucent linear structures parallel to the spicules, and (5) the absence of a palpable lesion or skin changes (fig 1). The absence of macrocalcifications is also considered a feature.

"It is generally accepted that the term radial scar refers to lesions less than $1 \mathrm{~cm}$, whereas complex sclerosing lesion is used to describe lesions $1 \mathrm{~cm}$ or larger"

However, as many groups have demonstrated, ${ }^{32-21}$ none of these findings is specific and similar features may be seen in carcinomas. It has been emphasised that calcifications may occur in RS, ${ }^{21}$ being the only abnormality in some cases. ${ }^{12}{ }^{14}$ In addition, not all demonstrate a radiolucent centre. Ultrasonography fares no better in the differentiation of RS from malignant lesions. ${ }^{22}$ The notion that RS are impalpable has been challenged, and Wallis et al recorded that six of 24 cases in a screening programme presented with clinical masses. ${ }^{19}$

\section{PATHOLOGY}

Grossly, RS lesions may mimic carcinoma because of their stellate configuration with central puckering and cream/yellow elastotic flecks, in addition to their firm texture. ${ }^{23}$ Histologically, RSs are pseudo-infiltrative lesions, the appearance of which may vary according to the plane of section or the stage of evolution. Classically, they are characterised by a fibroelastotic core with entrapped ducts demonstrating a dual myoepithelial and epithelial layer, with surrounding radiating

Abbreviations: DCIS, ductal carcinoma in situ; ER , oestrogen receptor; RS, radial scar 


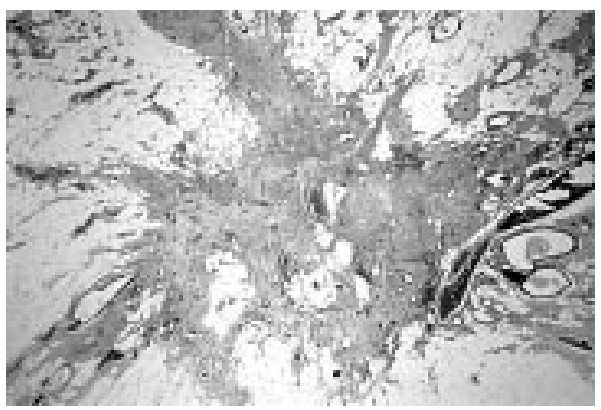

Figure 2 Low power view of radial scar with central fibroelastotic core and radiating ducts (haematoxylin and eosin stained).

ducts and lobules exhibiting variable epithelial hyperplasia, duct ectasia, adenosis, and papillomatosis ${ }^{23}$ (figs 2 and 3). Calcification is common, ${ }^{57924}$ and is found in areas of adenosis or epithelial hyperplasia. Lesions may be multifocal and bilateral, ${ }^{679102526}$ and "clustering" of scars may occur. ${ }^{25}$ Benign changes such as cysts, sclerosing adenosis, and usual type hyperplasia are frequently found in the residual breast. ${ }^{27}$ It is generally accepted that the term RS refers to lesions less than $1 \mathrm{~cm}$, whereas complex sclerosing lesion is used to describe lesions $1 \mathrm{~cm}$ or larger. ${ }^{28}$ Although epithelial atypia is not a diagnostic feature, these lesions are not infrequently associated with atypical hyperplasia (ductal or lobular) and in situ or invasive carcinoma. ${ }^{131618242729-32}$ In addition, the pseudo-infiltrative appearance renders distinction from infiltrating carcinomas (especially tubular carcinoma) difficult in some cases; this is particularly challenging if there is superimposed in situ neoplasia. ${ }^{33}$ The demonstration of a myoepithelial component by smooth muscle actins or calponin may assist in this situation. ${ }^{33}{ }^{34}$ Gobbi et al have emphasised that a minority may demonstrate perineural infiltration by benign ducts and caution against using this as a sole criterion of malignancy ${ }^{35}$ (fig 3).

\section{PATHOGENESIS}

The pathogenesis of RS remains obscure. It has been postulated that these lesions arise as a result of unknown injury, leading to fibrosis and retraction of surrounding breast tissue, thus imparting a stellate configuration. Associations with duct ectasia and duct obliteration have also been suggested, ${ }^{23}$ in addition to a chronic inflammatory response. ${ }^{25}$ However, central duct obliteration and elastosis may also be seen in carcinomas. ${ }^{36}$ Battersby and Anderson performed an ultrastructural study of RS derived from 38 patients $^{37}$; they defined an initial "cellular" phase characterised by abundant central myofibroblasts, followed by a later "mature" phase with few myofibroblasts, prominent elastic/collagen fibres, and parenchymal distortion. In another study, ${ }^{38}$ microvessel density was increased in a subset of RS lesions at the periphery, with reduced vascularity in the central "older" fibrotic zone. There does not appear to be an association with menopausal status, parity, or contraceptive use, ${ }^{7259}$ or with cytotoxic/tamoxifen treatment. ${ }^{39}$

It has also been hypothesised that there is a derangement of the normal stromal/epithelial interaction in RS. In an mRNA study of nine cases of RS, ${ }^{38}$ a variety of vascular stromal factors were examined, including collagen type l, fibronectin, thrombospondin 1, vascular permeability factor/vascular endothelial growth factor and its receptor KDR. Interestingly, a similar pattern of expression was documented in four control breast carcinomas, although expression was generally focal and less intense in RS. In a separate study, Iqbal and co-workers looked at allelic imbalance (chromosomes $16 \mathrm{q}$ and $8 \mathrm{p}$ ) in addition to both oestrogen receptor $\alpha$ (ER- $\alpha)$ and Ki67 expression by means of immunohistochemistry. ${ }^{40}$ They showed some evidence of ER dysregulation with respect to Ki67 within hyperplastic areas of RS, but found no significant allelic imbalance compared with background breast.

\section{BIOLOGICAL RELEVANCE}

Over the years there has been considerable debate as to whether RS is: (1) an incidental, non-relevant finding, (2) a direct precursor of carcinoma, or (3) a marker of neoplastic risk. In Wellings and Alpers's detailed necropsy based study of 83 random cases compared with 107 cancer associated breasts, RS was a not infrequent microscopic finding, being found in $14 \%$ and $26 \%$, respectively. ${ }^{6}$ These authors used a subgross technique and noted that the average number of RS lesions was higher in the malignant group; they suggested that RS was a risk factor for carcinoma, rather than being an obligate precursor lesion. In an additional necropsy study of 84 patients with breast cancer, it was recorded that RS was more likely to occur in areas of benign breast change, usually being present in the contralateral breast to carcinoma. ${ }^{39}$ In contrast,
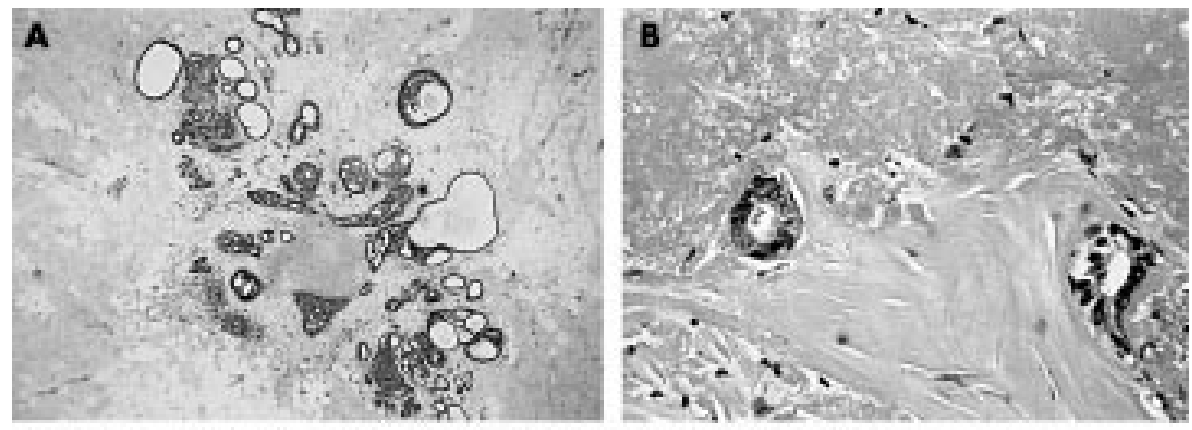

Figure 3 (A) Small radial scar (RS) demonstrating an elastotic centre with a corona of hyperplastic ducts and lobules; (B) central atrophic ducts in an elastotic stroma; (C) medium power view of ducts showing epithelial hyperplasia; (D) perineural invasion at the edge of a benign RS (haematoxylin and eosin stained).
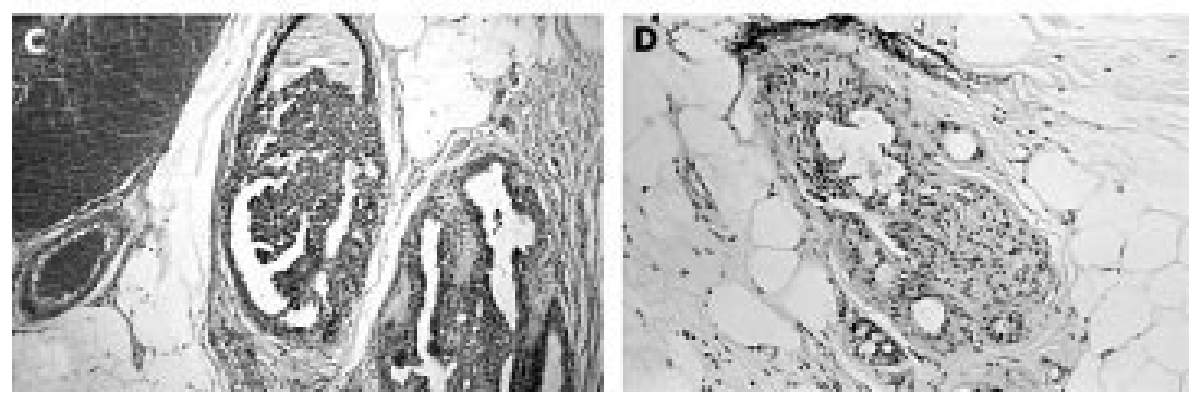
Linell et al hypothesised that because some RS lesions showed "transitional features" of tubular carcinomas, they represented direct precursor lesions, ${ }^{26}$ as did Fisher et al, who regarded RS as "incipient" tubular carcinoma. ${ }^{5}$ Other groups have furthered this claim on the basis of hyaluran expression in the stroma of $\mathrm{RS}^{41}$ and image analysis data. ${ }^{42}$

\section{"There does not appear to be an association with menopausal status, parity, or contraceptive use, or with cytotoxic/tamoxifen treatment"}

In recent years, accumulating evidence challenges this last view and it is likely that breast carcinomas produce a stromal reaction which mimics $\mathrm{RS}^{27}{ }^{36}$ Although RS lesions are regarded as benign, it is becoming increasingly clear that they are associated with atypia and/or malignancy on histology in a significant number of cases. In some series, malignancy rates of up to 30\% have been recorded. ${ }^{13} 1824272932$ In one small study of core biopsy confirmed RS, it was documented that ductal carcinoma in situ (DCIS) or infiltrating carcinoma was present in two of five cases, ${ }^{29}$ whereas in the study of Frouge et al on 40 patients with mammographic RS, half were malignant, eight showing evidence of RS on histology. ${ }^{18}$ However, as has been suggested, high malignancy rates may be a reflection of small series size, ${ }^{30}$ or more likely referral bias. ${ }^{30}{ }^{33}$ In support of this, Cawson and colleagues recorded a 7\% incidence of DCIS in RS in a screened population with no invasive carcinomas. ${ }^{30}$ In their definitive histological study of 126 lesions derived from 91 patients, Sloane et al found that the risk of malignancy was related to lesion size (being uncommon in RS $<6-7 \mathrm{~mm}$ ), patient age (malignancy was not demonstrated in patients under 40), and method of detection (usually screening). ${ }^{27}$ This last factor is probably a function of lesion size and $\operatorname{age}^{27}$; indeed evidence from the literature indicates that most patients present in the 40-60 age group. It has also been suggested that there is an increased incidence of metaplastic carcinoma in complex sclerosing lesions. ${ }^{43}$

In a longterm study (with a median follow up of 12 years) arising from the Nurses' Health Study, in which almost 1400 cases of open biopsies for benign breast disease were examined, Jacobs et al found that the presence of RS conferred double the risk of developing subsequent malignancy, regardless of the type of primary breast disease ${ }^{10}$ They recorded that RS was a not infrequent incidental finding (7.1\%), and that the increased risk was conferred on both breasts. Importantly, the risk of developing cancer was higher in those with increasing RS size and number. They found no association with age at menarche, parity, age at birth of first child, or body mass index, although women with subsequent malignancy were more likely to have had a positive family history. The evidence suggests that in most cases, low grade carcinomas (both in situ and invasive), with a good prognosis, occur. ${ }^{18} 24273233$

\section{SCREENING MAMMOGRAPHY AND PATIENT MANAGEMENT}

Undoubtedly, the incidence of RS has increased dramatically as a result of population based screening programmes with documented rates of $0.03-0.09 \% .^{11} 12141930$ Spencer et al, in a study of 108 benign lesions removed during the prevalent round of a screening programme, found 18 RS lesions, which presented as either non-comedo type suspicious calcifications or more frequently as architectural distortion. ${ }^{44}$ Burnett and colleagues recorded that RS constituted 23 of 137 benign lesions, of which four harboured atypical hyperplasia; they also demonstrated that microcalcifications may be the only mammographic abnormality. ${ }^{14}$ In an additional study of 80 screen detected R4 spiculated lesions, there were 46 RS lesions (eight with DCIS). ${ }^{24}$

Because RS cannot be reliably differentiated from carcinoma using radiographic modalities it has been recommended that surgical excision should be performed on all cases. Recently, however, some groups ${ }^{13} 16{ }^{31}$ have argued that thorough core biopsy evaluation in conjunction with careful mammographic surveillance and larger tissue samples (for example, vacuum assisted sampling, mammotome) ${ }^{13} 1645$ may be appropriate in selected subsets of patients. Two recent publications $^{13}{ }^{30}$ have suggested that core needle biopsy diagnosis is probably reliable in RS when there is no associated atypical hyperplasia, a minimum of five $^{30}$ to 12 core specimens ${ }^{13}$ are evaluated, and the mammographic findings are concordant. Similarly, Kirwan et al, ${ }^{31}$ using 14 gauge stereotactic biopsies in 72 stellate lesions (including 34 RSs that proceeded to open biopsy), found that in the absence of atypia on the needle core, a final diagnosis of malignancy was unlikely. They achieved an absolute sensitivity of $78 \%$ for the detection of malignancy when multiple cores were used, with a positive predictive value of $100 \%$.

"Undoubtedly, the incidence of radial scar has increased dramatically as a result of population based screening programmes with documented rates of 0.03 $0.09 \% "$

We routinely perform needle core biopsies on our cases of suspected mammographic RS (unpublished data, 2003) and it is our screening programme policy to excise all such lesions. In line with other groups ${ }^{30}{ }^{31}$ we have found that a preoperative diagnosis is possible in over $85 \%$ of cases. When these are stratified according to histologically confirmed RS versus RS mimics, higher diagnosis rates can be achieved in the last group. This is of particular value where the core yields an unequivocal diagnosis of malignancy because it allows definitive surgical treatment at the first operation. We emphasise, in line with others, ${ }^{30}$ the importance of adequately sampling the periphery of the lesion, in addition to the centre, because carcinoma (both in situ and invasive) is more likely to develop at the periphery. ${ }^{27}$ The value of cytology in the preoperative assessment of RS appears to be limited. ${ }^{24} 3246$

The detection of incidental microscopic RS on needle core biopsies for microcalcifications is more problematic. Because the likelihood of detecting carcinoma is a function of lesion size ${ }^{27}$ it is probable that in the absence of a radiological mass lesion or architectural distortion, a malignant outcome is less likely. Indeed, Philpotts et al documented that of their RS cases (there were no malignancies), $44 \%$ presented with calcification. $^{16}$ However, the findings of Jacobs and colleagues $^{10}$ suggest that at least close clinical surveillance is warranted, analogous to patient follow up for a diagnosis of atypical hyperplasia. In line with this, the studies of both Philpotts and colleagues ${ }^{16}$ and Cawson and colleagues ${ }^{30}$ showed high atypia rates. Importantly, in the screening based study of Cawson et al, ${ }^{30} 57 \%$ of the RS lesions demonstrated atypical ductal hyperplasia at excision, ${ }^{30}$ although this has not been our experience (personal observation, 2003).

\section{CONCLUSION}

The detection of RS has increased dramatically with the introduction of population based mammographic screening programmes. Because currently we cannot reliably exclude malignancy in RS, and in light of the high atypia rate documented by some groups, ${ }^{16}{ }^{30}$ it is our opinion that excision biopsy continues to be justified. Nevertheless, we acknowledge that there is an accumulating body of evidence to support close mammographic surveillance in conjunction with thorough core biopsy evaluation in selected patients; this does require further evaluation with larger patient numbers (preferably in a screening setting). 
Take home messages

- Radial scar (RS) is a benign, well recognised, radiological and pathological entity

- Postmortem studies indicate that these lesions are common, especially in association with benign breast disease

- The introduction of population based screening programmes has provided evidence that RS is associated with atypia and/or malignancy. RS may be an independent risk factor for the development of carcinoma in either breast

- In view of this association, excision biopsy is justified in RS, although it has been argued that core biopsy evaluation and surveillance may be appropriate in selected patients, but further evaluation with larger patient numbers is needed

\section{ACKNOWLEDGEMENTS}

We are indebted to Ms F Grehan for photographic assistance.

\section{Authors' affiliations}

M Kennedy, Department of Pathology, The National Breast Screening Programme Eccles Unit and Mater Misericordiae University Hospital, Eccles St, Dublin 7, Ireland

A V Masterson, F Flanagan, Department of Radiology, The National Breast Screening Programme Eccles Unit and Mater Misericordiae University Hospital

M Kerin, Department of Surgery, The National Breast Screening

Programme Eccles Unit and Mater Misericordiae University Hospital

\section{REFERENCES}

1 Fenoglio C, Lattes R. Sclerosing papillary proliferations in the female breast. A benign lesion often mistaken for carcinoma. Cancer 1974;33:691-700.

2 Azzopardi JG. Overdiagnosis of malignancy. In: Azzopardi JG, ed. Problems in breast pathology. Philadelphia: WB Saunders, 1979:167-91.

3 Rickert RR, Kalisher L, Hutter RVP. Indurative mastopathy: a benign sclerosing lesion of breast with elastosis which may simulate carcinoma. Cancer 1981;47:561-71.

4 Tremblay G, Buell RH, Seemayer TA. Elastosis in benign sclerosing ductal proliferation of the female breast. Am J Surg Pathol 1977; 1:155-9.

5 Fisher ER, Palekar AS, Kotwal N, et al. A nonencapsulated sclerosing lesion of the breast. Am J Clin Pathol 1979;71:240-6.

6 Wellings SR, Alpers CE. Subgross pathologic features and incidence of radial scars in the breast. Hum Pathol 1984;15:475-9.

7 Nielsen $\mathbf{M}$, Jensen J, Andersen JA. An autopsy study of radial scar in the female breast. Histopathology 1985;9:287-95

8 Bhathal PS, Brown RW, Lesueur GC. Frequency of benign and malignant breast lesions in 207 consecutive autopsies in Australian women. Br J Cancer 1985:51:271-8.

9 Andersen JA, Gram JB. Radial scar in the female breast. A long-term follow-up study of 32 cases. Cancer 1984;53:2557-60.

10 Jacobs TW, Byrne C, Colditz G, et al. Radial scars in benign breast-biopsy specimens and the risk of breast cancer. N Engl J Med 1999:340:430-6.

11 Tabar L, Dean PB. Stellate lesions. In: Tabar L, Dean PB, eds. Teaching atlas of mammography, Second revised edition. New York: Georg Thieme Verlag, 1985:87-136

12 Patel A, Steel Y, McKenzie J, et al. Radial scars: a review of 30 cases. Eur J Surg Oncol 1997;23:202-5

13 Brenner RJ, Jackman RJ, Parker SH, et al. Percutaneous core needle biopsy of radial scars of the breast: when is excision necessary? Am J Roentgenol 2002;179:1179-84

14 Burnett SJD, Ng YY, Perry NM, et al. Benign biopsies in the prevalent round of breast screening: a review of 137 cases. Clin Radiol 1995;50:254-8

15 Ciatto S, Morrone D, Catarzi S, et al. Radial scars of the breast: review of 38 consecutive mammographic diagnoses. Radiology $1993 ; 187: 757-60$
16 Philpotts LE, Shaheen NA, Jain KS, et al. Uncommon high-risk lesions of the breast diagnosed at stereotactic core-needle biopsy: clinical importance. Radiology 2000;216:831-7.

17 Alleva DQ, Smetherman DH, Farr GH, et al. Radial scar of the breast: radiologic-pathologic correlation in 22 cases. Radiographics 1999; 19:S27-35

18 Frouge C, Tristant H, Guinebretiere JM, et al. Mammographic lesions suggestive of radial scars: microscopic findings in 40 cases. Radiology 1995:195:623-5

19 Wallis MG, Devakumar R, Hosie KB, et al. Complex sclerosing lesions (radial scars) of the breast can be palpable. Clin Radiol 1993;48:319-20.

20 King TA, Scharfenberg JC, Smetherman DH, et al. A better understanding of the term radial scar. Am J Surg 2000;180:428-33.

21 Orel SG, Evers K, Yeh I-T, et al. Radial scar with microcalcifications: radiologic-pathologic correlation. Radiology 1992;183:479-82.

22 Cohen MA, Sferlazza SJ. Role of sonography in evaluation of radial scars of the breast. Am J Roentgenol 2000;174:1075-8.

23 Elston CW, Ellis IO, Goulding H. Sclerosing lesions. In: Elston CW, Ellis $1 \mathrm{O}$, eds. The breast. Systemic pathology, Vol. 13, 3rd ed. Edinburgh: Churchill Livingstone, 1998:107-31.

24 Douglas-Jones AG, Pace DP. Pathology of R4 spiculated lesions in the breast screening programme. Histopathology 1997;30:214-20.

25 Anderson TJ, Battersby S. Radial scars of benign and malignant breasts: comparative features and significance. J Pathol 1985; 147:23-32.

26 Linell $\mathbf{F}$, Ljungberg $O$, Andersson I. Breast carcinoma: aspects of early stages, progression and related problems. Acta Pathol Microbiol Scand Supp/ 1980;272:14-62

27 Sloane JP, Mayers MM. Carcinoma and atypical hyperplasia in radial scars and complex sclerosing lesions: importance of lesion size and patient age. Histopathology 1993;23:225-31

28 Page DL, Anderson TJ. Radial scars and complex sclerosing lesions. In: Page DL, Anderson TJ, eds. Diagnostic histopathology of the breast. Edinburgh: Churchill Livingstone, 1987:89-103.

29 Jackman RJ, Nowels KW, Rodriguez-Soto J, et al. Stereotactic, automated, large-core needle biopsy of non-palpable breast lesions: false negative and histologic underestimation rates after long-term follow-up. Radiology 1999;210:799-805.

30 Cawson JN, Malara F, Kavanagh A, et al. Fourteen-gauge needle core biopsy of mammographically evident radial scars. Is excision necessary? Cancer 2003:97:345-51.

31 Kirwan SE, Denton ERE, Nash RM, et al. Multiple 14G stereotactic core biopsies in the diagnosis of mammographically detected stellate lesions of the breast. Clin Radiol 2000;55:763-6

32 Mokbel K, Price RK, Mostafa A, et al. Radial scar and carcinoma of the breast: microscopic findings in 32 cases. Breast 1999;8:339-42.

33 Alvarado-Cabrero I, Tavassoli FA. Neoplastic and malignant lesions involving or arising in a radial scar: a clinicopathologic analysis of 17 cases. Breast J 2000;6:96-102.

34 Yaziji H, Gown AM, Sneige N. Detection of stromal invasion in breast cancer: the myoepithelial markers. Adv Anat Pathol 2000;7:100-9.

35 Gobbi $\mathbf{H}$, Jensen RA, Simpson JF, et al. Atypical ductal hyperplasia and ductal carcinoma in situ of the breast associated with perineural invasion. Hum Pathol 2001·32:785-90.

36 Egger $\mathbf{H}$, Dressler W. A contribution to the natural history of breast cancer. I. Duct obliteration with periductal elastosis in the centre of breast cancers. Arch Gynecol 1982;231:191-8.

37 Battersby S, Anderson TJ. Myofibroblast activity of radial scars. J Pathol 1985; 147:33-40.

38 Jacobs TW, Schnitt SJ, Tan X, et al. Radial scars of the breast and breas carcinomas have similar alterations in expression of factors involved in vascular stroma formation. Hum Pathol 2002;33:29-38.

39 Nielsen M, Christensen L, Andersen J. Radial scars in women with breast cancer. Cancer 1987;59:1019-25.

40 Iqbal M, Shoker BS, Foster CS, et al. Molecular and genetic abnormalities in radial scar. Hum Pathol 2002:33:715-22.

41 De La Torre M, Wells AF, Bergh J, et al. Localization of hyaluronan in normal breast tissue, radial scar and tubular breast carcinoma. Hum Pathol 1993;24:1294-7.

42 Ruiz-Sauri A, Almenar-Medina S, Callaghan RC, et al. Radial scar versus tubular carcinoma of the breast. A comparative study with quantitative techniques (morphometry, image- and flow cytometry). Pathol Res Pract 1995:191:547-54.

43 Denley H, Pinder SE, Tan PH, et al. Metaplastic carcinoma of the breast arising within complex sclerosing lesion: a report of five cases. Histopathology 2000;36:203-9

44 Spencer NJB, Evans AJ, Galea M, et al. Pathological-radiological correlations in benign lesions excised during a breast screening programme. Clin Radiol 1994:49:853-6.

45 Carder PJ, Liston JC. Will the spectrum of lesions prompting a "B3" breast core biopsy increase the benign biopsy rate? J Clin Pathol 2003;56: 133-8.

46 Orell SR. Radial scar/complex sclerosing lesion-a problem in the diagnostic work-up of screen-detected breast lesions. Cytopathology 1999:10:250-8. 